\title{
Muonic hydrogen and the proton size
}

\author{
Wayne W. Repko ${ }^{1, *}$ and Duane A. Dicus ${ }^{2, \dagger}$ \\ ${ }^{1}$ Department of Physics and Astronomy, Michigan State University, East Lansing, Michigan 48824, USA \\ ${ }^{2}$ Department of Physics, University of Texas, Austin, Texas 78712, USA
}

(Received 9 November 2017; revised manuscript received 21 June 2018; published 6 July 2018)

\begin{abstract}
We reexamine the structure of the $n=2$ levels of muonic hydrogen using a two-body potential that includes all relativistic, recoil and one-loop corrections. The potential was originally derived from QED to describe the muonium atom and accounts for all contributions to order $\alpha^{5}$. Since one-loop corrections are included, the anomalous magnetic moment contributions of the muon can be identified and replaced by the proton anomalous magnetic moment to describe muonic hydrogen with a pointlike proton. This serves as a convenient starting point to include the dominant electron vacuum polarization corrections to the spectrum and extract the proton's mean squared radius $r_{p}=\sqrt{\left\langle r^{2}\right\rangle}$. Our results are consistent with other theoretical calculations that find that the muonic hydrogen value for $r_{p}$ is smaller than the result obtained from electron scattering.
\end{abstract}

DOI: 10.1103/PhysRevD.98.013002

\section{INTRODUCTION}

The muonic hydrogen experiments [1,2] measure both the $2^{3} S_{1 / 2} \leftrightarrow 2^{5} P_{3 / 2}$ and the $2^{1} S_{1 / 2} \leftrightarrow 2^{3} P_{3 / 2}$ transitions. The experimental results are, respectively, $\quad 49881.35(65) \mathrm{GHz}=206.2925(3) \mathrm{meV}$ and $54611.16(1.05) \mathrm{GHz}=225.8535(4) \mathrm{meV}$. These measurements have been compared with a variety of theoretical calculations [3-9] that include a dependence on the mean squared proton radius $\left\langle r^{2}\right\rangle$. Our purpose here is to compare the contributions to the Lamb shift that are independent of the proton structure with those of previous calculations. We find a value of $205.980 \mathrm{meV}$, about $0.07 \mathrm{meV}$ smaller than other theoretical calculations.

If proton structure corrections are included, the resulting values of $r_{p}$ from muonic hydrogen are systematically smaller than those generally obtained from electron scattering data [10], leading to a disparity between the two approaches. Some of the disparity could be associated with uncertainties in the scattering data. The proton radius experiment at the Jefferson Laboratory is designed to address this issue [11]. A recent spectroscopic measurement of the Rydberg constant [12] reports a smaller value of $r_{p}$ consistent with muonic hydrogen, but a new spectroscopic measurement of the $1 S \rightarrow 3 S$ transition in hydrogen

\footnotetext{
repko@pa.msu.edu

†dicus@physics.utexas.edu
}

Published by the American Physical Society under the terms of the Creative Commons Attribution 4.0 International license. Further distribution of this work must maintain attribution to the author(s) and the published article's title, journal citation, and DOI. Funded by SCOAP ${ }^{3}$.
[13] supports a larger value as do most other spectroscopic measurements.

Here, we reexamine the theoretical calculation from a slightly different starting point. Our approach is to modify the two-body potential originally derived from QED to describe the muonium atom [14]. This potential contains all relativistic, recoil and one-loop terms that contribute to order $\alpha^{5}$. The inclusion of the one-loop corrections enables us to identify the muon anomalous magnetic moment and replace it by the proton's anomalous magnetic moment $\kappa=1.79285$. The resulting potential can be used to calculate the fine structure, hyperfine structure, Lamb shift and recoil corrections for muonic hydrogen with a a pointlike proton. It also serves as the starting point to include the dominant electron vacuum polarization contributions. The resulting hyperfine, spin-orbit, tensor and spin-independent potentials are [15], respectively,

$$
\begin{aligned}
V_{H F}= & \frac{4 \pi \alpha}{m_{1} m_{2}}\left[\frac{2}{3}\left(1+a_{\mu}\right)(1+\kappa)+\frac{\alpha}{\pi} \frac{m_{1} m_{2}}{m_{1}^{2}-m_{2}^{2}} \ln \left(\frac{m_{2}^{2}}{m_{1}^{2}}\right)\right] \\
& \times \vec{S}_{1} \cdot \vec{S}_{2} \delta(\vec{r}) \\
V_{L S}= & \frac{\alpha}{r^{3}}\left[\frac{\left(1+2 a_{\mu}\right)}{2 m_{1}^{2}}+\frac{\left(1+a_{\mu}\right)}{m_{1} m_{2}}\right] \vec{L} \cdot \vec{S}_{1} \\
& +\frac{\alpha}{r^{3}}\left[\frac{(1+2 \kappa)}{2 m_{2}^{2}}+\frac{(1+\kappa)}{m_{1} m_{2}}\right] \vec{L} \cdot \vec{S}_{2} \\
V_{T}= & \frac{\alpha\left(1+a_{\mu}\right)(1+\kappa)}{m_{1} m_{2} r^{3}}\left(3 \vec{S}_{1} \cdot \hat{r} \vec{S}_{2} \cdot \hat{r}-\vec{S}_{1} \cdot \vec{S}_{2}\right)
\end{aligned}
$$




$$
\begin{aligned}
V_{S I}= & -\frac{\alpha}{m_{1} m_{2} r} \vec{p}^{2}+\frac{\alpha \pi}{2 \mu^{2}} \delta(\vec{r})-\frac{1}{8}\left(\frac{1}{m_{1}^{3}}+\frac{1}{m_{2}^{3}}\right)\left(\vec{p}^{2}\right)^{2}+\frac{\alpha^{2} \mu}{2 m_{1} m_{2} r^{2}} \\
& +\alpha^{2}\left[\frac{4}{3}\left(\frac{1}{\mu^{2}} \ln \left(\frac{\mu}{\lambda_{I F}}\right)-\frac{1}{m_{1}^{2}} \ln \left(\eta_{2}\right)-\frac{1}{m_{2}^{2}} \ln \left(\eta_{1}\right)\right) \delta(\vec{r})+\left(\frac{2\left(m_{1}^{2} \ln \left(\eta_{1}\right)-m_{2}^{2} \ln \left(\eta_{2}\right)\right)}{m_{1} m_{2}\left(m_{1}^{2}-m_{2}^{2}\right)}\right) \delta(\vec{r})\right. \\
& \left.+\frac{7}{6 \pi m_{1} m_{2}} \nabla^{2}\left(\frac{\ln (\mu r)+\gamma}{r}\right)-\frac{4}{15}\left(\frac{1}{m_{1}^{2}}+\frac{1}{m_{2}^{2}}\right) \delta(\vec{r})\right]
\end{aligned}
$$

Here, $\mu$ is the reduced mass, $\eta_{i}=m_{i} /\left(m_{1}+m_{2}\right), \gamma$ is Euler's constant and $\lambda_{I F}$ is the infrared cutoff. The last term in Eq. (4) is the contribution from the muon and proton vacuum polarizations. In what follows, we use [16] $m_{1}=105.6583715(35) \mathrm{MeV}, m_{2}=938.272046(21) \mathrm{MeV}$, $\mu=94.9645 \mathrm{MeV}, \quad m_{e}=0.510998928(11) \mathrm{MeV}, \quad \alpha=$ $1 / 137.035999074(44)$ and $a_{\mu}=\alpha / 2 \pi$.

Much of the reason for undertaking the following calculation is that Eq. (4) contains several terms that differ from those commonly used in determining the muonic hydrogen spectrum. In particular, the order $\alpha$ delta function term behaves as $\mu^{-2}$ whereas the corresponding term in [5] has an overall $\left(1 / m_{1}^{2}+1 / m_{2}^{2}\right)$ factor. The difference in the $s$-state contribution is several meV in the order $\alpha^{4}$ correction. Also, the one-loop term $\mu \alpha^{2} /\left(2 m_{1} m_{2} r^{2}\right)$ contributes at order $\alpha^{4}$ at the few meV level. The $\mu^{-2}$ dependence of the order $\alpha^{2}$ one-loop $\ln \left(\mu / \lambda_{I F}\right)$ term results in a recoil correction to the Lamb shift of order $\mu^{3} \alpha^{5} / m_{2}^{2}$ that is larger than the order $\alpha^{6}$ correction to this contribution given in [5]. We undertook the calculation to determine the implication of these differences.

\section{ELECTRON VACUUM POLARIZATION EFFECTS}

\section{A. One-loop correction to the Coulomb potential}

The dominant contributions to the $2 P-2 S$ splitting in muonic hydrogen are due to the electron vacuum polarization corrections to the photon propagator. These contributions can be included by using the dispersion representation for the photon propagator [17-19]

$$
D\left(k^{2}\right)=\frac{1}{k^{2}}-\int_{0}^{\infty} \frac{d \lambda}{\lambda} \frac{\Delta(\lambda)}{\lambda-k^{2}},
$$

where $\Delta\left(q^{2}\right)$ is

$$
\Delta\left(q^{2}\right)=\frac{(2 \pi)^{3}}{3 q^{2}} \sum_{n} \delta^{(4)}\left(q-q_{n}\right)\left\langle 0\left|j_{\mu}(0)\right| n\right\rangle\left\langle n\left|j^{\mu}(0)\right| 0\right\rangle
$$

For the $e \bar{e}$ intermediate state, $\Delta^{(2)}(\lambda)$ is

$$
\Delta^{(2)}(\lambda)=\frac{\alpha}{3 \pi}\left(1+2 m_{e}^{2} / \lambda\right) \sqrt{1-4 m_{e}^{2} / \lambda} \theta\left(\lambda-4 m_{e}^{2}\right) .
$$

If we take $k^{2}$ to be spacelike, then the modified Coulomb interaction at the one-loop level is

$$
\begin{aligned}
V\left(\vec{k}^{2}\right) & =-\frac{e^{2}}{\vec{k}^{2}}-e^{2} \int_{4 m_{e}^{2}}^{\infty} \frac{d \lambda}{\lambda} \frac{\Delta^{(2)}(\lambda)}{\vec{k}^{2}+\lambda} \\
& =V_{C}\left(\vec{k}^{2}\right)+V_{V P}\left(\vec{k}^{2}\right) .
\end{aligned}
$$

The explicit form of $V_{V P}\left(\vec{k}^{2}\right)$ in momentum space can be obtained by integrating over $\lambda$ which results in

$$
V_{V P}\left(\vec{k}^{2}\right)=\frac{-e^{2}}{\vec{k}^{2}} \Pi_{f}^{(2)}\left(\vec{k}^{2}\right)
$$

with

$$
\begin{aligned}
\Pi_{f}^{(2)}\left(\vec{k}^{2}\right)= & \frac{2 \alpha}{\pi}\left[\frac{1}{3}\left(1-2 m_{e}^{2} / \vec{k}^{2}\right)\right. \\
& \left.\times\left(\sqrt{1+4 m_{e}^{2} / \vec{k}^{2}} \operatorname{arcsinh}\left(\frac{|\vec{k}|}{2 m_{e}}\right)-1\right)+\frac{1}{18}\right] .
\end{aligned}
$$

$\Pi_{f}^{(2)}\left(\vec{k}^{2}\right)$ is the electron one-loop vacuum polarization correction in the spacelike region.

Transforming to coordinate space

$$
\begin{aligned}
& V_{V P}(\vec{r})=\frac{1}{(2 \pi)^{3}} \int d^{3} k V_{V P}(\vec{k}) e^{i \vec{k} \cdot \vec{r}}, \\
& V_{V P}(\vec{r})=-\frac{\alpha}{r} \int_{4 m_{e}^{2}}^{\infty} \frac{d \lambda}{\lambda} \Delta^{(2)}(\lambda) e^{-\sqrt{\lambda} r} .
\end{aligned}
$$

To compute the effect of $V_{V P}$, we need to calculate the difference between $\left\langle 21 m\left|V_{V P}\right| 21 m\right\rangle$ and $\left\langle 200\left|V_{V P}\right| 200\right\rangle$. For example, $\left\langle 21 m\left|V_{V P}\right| 21 m\right\rangle$ is

$$
\begin{aligned}
& \left\langle 21 m\left|V_{V P}\right| 21 m\right\rangle=-\frac{\alpha}{24 a} \int_{4 m_{e}^{2}}^{\infty} \frac{d \lambda}{\lambda} \Delta^{(2)}(\lambda) \int_{0}^{\infty} d t t^{3} e^{-(1+a \sqrt{\lambda}) t}, \\
& \left\langle 21 m\left|V_{V P}\right| 21 m\right\rangle=-\frac{\alpha}{4 a} \int_{4}^{\infty} \frac{d x}{x} \frac{\Delta^{(2)}(x)}{\left(1+m_{e} a \sqrt{x}\right)^{4}}
\end{aligned}
$$

with $a=1 / \mu \alpha$ and $\lambda=m_{e}^{2} x$. A similar calculation gives 
$\left\langle 200\left|V_{V P}\right| 200\right\rangle=-\frac{\alpha}{4 a} \int_{4}^{\infty} \frac{d x}{x} \frac{\Delta^{(2)}(x)\left(1+2 m_{e}^{2} a^{2} x\right)}{\left(1+m_{e} a \sqrt{x}\right)^{4}}$.

The difference between Eqs. (14) and (15) is

$$
\begin{aligned}
E E_{V P} & =\Delta(2 P-2 S)=\frac{\mu \alpha^{3}}{6 \pi} \int_{4}^{\infty} \frac{d x(1+2 / x) \sqrt{1-4 / x} m_{e}^{2} a^{2}}{\left(1+m_{e} a \sqrt{x}\right)^{4}} \\
& =205.007 \mathrm{meV} .
\end{aligned}
$$

This agrees with [5]. It is worth noting that the muon contribution from Eq. (16) is $0.0167 \mathrm{meV}$ which is virtually the same as the $0.0168 \mathrm{meV}$ result coming from the $m_{1}^{-2}$ muon contribution in the last term of Eq. (4). The proton vacuum contribution from the $m_{2}^{-2}$ term in Eq. (4) is indistinguishable from the result obtained using Eq. (16).

\section{B. Two-loop correction to the Coulomb potential}

The two-loop contributions to $\Delta(\lambda), \Delta^{(4)}(\lambda)$, have been calculated by Källen and Sabry [19]. They contain both the reducible double electron bubble diagram and the irreducible fourth-order term $\Pi_{f}^{(4)}\left(\vec{k}^{2}\right)$. These corrections can be expressed as a correction to the Coulomb potential in the form [20]

$$
V_{V P 2}(r)=-\frac{\alpha}{r} F(r),
$$

where

$$
\begin{aligned}
F(r)= & -\frac{\alpha^{2}}{\pi^{2}} \int_{1}^{\infty} d t e^{-2 \operatorname{tr}}\left[\left(\frac{13}{54 t^{2}}+\frac{7}{108 t^{4}}+\frac{2}{9 t^{6}}\right) \sqrt{t^{2}-1}\right. \\
& +\left(\frac{4}{3 t^{2}}+\frac{2}{3 t^{4}}\right) \sqrt{t^{2}-1} \log \left(8 t\left(t^{2}-1\right)\right) \\
& +\left(-\frac{44}{9 t}+\frac{2}{3 t^{3}}+\frac{5}{4 t^{5}}+\frac{2}{9 t^{7}}\right) \operatorname{arccosh}(t) \\
& \left.+\left(-\frac{8}{3 t}+\frac{2}{3 t^{5}}\right) \int_{t}^{\infty} d x f(x)\right]
\end{aligned}
$$

with

$f(x)=\frac{3 x^{2}-1}{x\left(x^{2}-1\right)} \operatorname{arccosh}(x)-\frac{1}{\sqrt{x^{2}-1}} \log \left(8 x\left(x^{2}-1\right)\right)$.

By transforming this potential to momentum space and comparing it with Eq. (5), $\lambda$ can be identified with $4 t^{2}$ and the second-order energy shift expressed as

$E E_{V P 2}=-\frac{\mu \alpha^{4}}{4 \pi^{2}} \int_{4}^{\infty} d x \frac{\Delta^{(4)}(\sqrt{x} / 2) m_{e}^{2} a^{2}}{\left(1+m_{e} a \sqrt{x}\right)^{4}}=1.508 \mathrm{meV}$,

where $\Delta^{(4)}(x)$ is

$$
\begin{aligned}
\Delta^{(4)}(x)= & \left(\frac{13}{54 x}+\frac{7}{108 x^{3}}+\frac{2}{9 x^{5}}\right) \sqrt{x^{2}-1}+\left(\frac{4}{3 x}+\frac{2}{3 x^{3}}\right) \sqrt{x^{2}-1} \log \left(8 x\left(x^{2}-1\right)\right)+\left(-\frac{44}{9}+\frac{2}{3 x^{2}}+\frac{5}{4 x^{4}}+\frac{2}{9 x^{6}}\right) \operatorname{arccosh}(x) \\
& +\left(-\frac{8}{3}+\frac{2}{3 x^{4}}\right)\left[\frac{2 \pi^{2}}{3}-\operatorname{arccosh}^{2}(x)-\log \left(8 x\left(x^{2}-1\right)\right) \operatorname{arccosh}(x)-2 \operatorname{Re}\left[\operatorname{Li}_{2}\left(\left(x+\sqrt{x^{2}-1}\right)^{2}\right)\right]\right. \\
& \left.+\operatorname{Li}_{2}\left(-\left(x-\sqrt{x^{2}-1}\right)^{2}\right)\right] .
\end{aligned}
$$

The terms in the large square brackets result from evaluating the last integral in Eq. (18) and $\operatorname{Li}_{2}(z)$ is the Spence function

$$
\operatorname{Li}_{2}(z)=-\int_{0}^{1} \frac{d t}{t} \log (1-z t)
$$

\section{Three-loop correction to the Coulomb potential}

The three-loop contribution to $\Delta(\lambda), \Delta^{(6)}(\lambda)$, is not available in the literature. These contributions have been calculated by Kinoshita and Nio [21]. They note that the three-loop correction is comprised of two reducible diagrams and one irreducible diagram that can be represented as

$$
\left(\Pi_{f}^{(2)}\left(\vec{k}^{2}\right)\right)^{3}+2 \Pi_{f}^{(2)}\left(\vec{k}^{2}\right) \Pi_{f}^{(4)}\left(\vec{k}^{2}\right)+\Pi_{f}^{(6)}\left(\vec{k}^{2}\right) .
$$

Remembering the reducible fourth-order dispersion result

$$
-e^{2} \int_{4 m_{e}^{2}}^{\infty} \frac{d \lambda}{\lambda} \frac{\Delta^{(4)}(\lambda)}{\vec{k}^{2}+\lambda}=-\frac{e^{2}}{\vec{k}^{2}} \Pi^{(4)}\left(\vec{k}^{2}\right)
$$

and

$$
\Pi^{(4)}\left(\vec{k}^{2}\right)=\left(\Pi_{f}^{(2)}\left(\vec{k}^{2}\right)\right)^{2}+\Pi_{f}^{(4)}\left(\vec{k}^{2}\right),
$$

it is possible to verify the contributions of the reducible terms. They are 0.000396 and $0.0029312 \mathrm{meV}$, respectively. The numerical evaluation of the $\Pi_{f}^{(6)}\left(\vec{k}^{2}\right)$ contribution is [21] $0.001103 \mathrm{meV}$, giving a total three-loop correction to the Coulomb potential of $0.004431 \mathrm{meV}$. 


\section{D. $\alpha^{4}$ second-order nonrelativistic perturbation correction}

The large size of the one-loop correction suggests that the contribution of $V_{V P}(\vec{r})$ in the second-order nonrelativistic perturbation theory is not negligible. Evaluating this correction necessitates using the radial portions of the Coulomb Green's function for $n=2$ and $\ell=0,1$, expressed as $\mu^{2} \alpha g_{2 \ell}\left(x, x^{\prime}\right)$. General expressions for these Green's functions were derived by Hostler [22,23] and explicit expressions for small values of $n$ are contained in $[24,25]$. Due to some typographical errors in the latter papers [ $g_{20}$ in Ref. [24] and Eq. (2.18) in Ref. [25]], the expressions for $g_{20}\left(x, x^{\prime}\right)$ and $g_{21}\left(x, x^{\prime}\right)(x=r / a$, $x^{\prime}=r^{\prime}(a)$ are given here:

$$
\begin{aligned}
g_{20}\left(x, x^{\prime}\right)= & e^{-\left(x+x^{\prime}\right) / 2}\left[( 2 - x ) ( 2 - x ^ { \prime } ) \left(\ln (x)+\ln \left(x^{\prime}\right)\right.\right. \\
& \left.+\frac{\left(x+x^{\prime}\right)}{4}+2 \gamma-\frac{15}{4}+\operatorname{Ei}\left(x_{<}\right)\right) \\
& +12-2 x-2 x^{\prime}-\frac{2}{x}-\frac{2}{x^{\prime}}+\frac{x}{x^{\prime}}+\frac{x^{\prime}}{x}-x x^{\prime} \\
& \left.+\left(2-x_{>}\right) e^{x_{<}}\left(\frac{1}{x_{<}}-1\right)\right] \frac{1}{4 \pi}, \\
g_{21}\left(x, x^{\prime}\right)= & \frac{x x^{\prime}}{3} e^{-\left(x+x^{\prime}\right) / 2}\left[\ln (x)+\ln \left(x^{\prime}\right)+\frac{\left(x+x^{\prime}\right)}{4}+2 \gamma\right. \\
& -\frac{49}{12}-\frac{3}{x}-\frac{3}{x^{2}}-\frac{2}{x^{3}}-\frac{3}{x^{\prime}}-\frac{3}{x^{\prime 2}}-\frac{2}{x^{\prime 3}}-\operatorname{Ei}\left(x_{<}\right) \\
& \left.+\left(\frac{1}{x_{<}}+\frac{1}{x_{<}^{2}}+\frac{2}{x_{<}^{3}}\right) e^{x_{<}}\right] \frac{3 \hat{x} \cdot \hat{x}^{\prime}}{4 \pi} .
\end{aligned}
$$

Here, $\operatorname{Ei}(x)$ is

$$
\operatorname{Ei}(x)=\int_{-\infty}^{x} d t \frac{e^{t}}{t}
$$

and

$$
\begin{aligned}
& x_{<}=x \theta\left(x^{\prime}-x\right)+x^{\prime} \theta\left(x-x^{\prime}\right), \\
& x_{>}=x^{\prime} \theta\left(x^{\prime}-x\right)+x \theta\left(x-x^{\prime}\right) .
\end{aligned}
$$

The contributions take the form

$\mu^{2} \alpha \int_{0}^{\infty} d r r^{2} R_{n \ell}(r) V_{V P}(r) \int_{0}^{\infty} d r^{\prime} r^{\prime 2} g_{n \ell}\left(x, x^{\prime}\right) V_{V P}\left(r^{\prime}\right) R_{n \ell}\left(r^{\prime}\right)$,

for $n=2$ and $\ell=0,1$. The integrations over $r, r^{\prime}$ can be evaluated exactly using Mathematica, and integrations over propagator parameters $\lambda$ and $\lambda^{\prime}$ can then be calculated numerically. The results are
$E E^{(2)} 2 P_{V P}=-0.0022671 \mathrm{meV}$ and $E E^{(2)} 2 S_{V P}=$ $-0.153164 \mathrm{meV}$ for a net contribution of [5]

$$
E E_{V P}^{(2)}=0.1509 \mathrm{meV}
$$

\section{E. $\alpha^{5}$ second-order nonrelativistic perturbation correction}

There is also a second-order nonrelativistic perturbative contribution from the combination of a one-loop vacuum polarization correction and a two-loop vacuum polarization correction. The calculation is similar to the one-loop second-order calculation and results in a $0.00215 \mathrm{meV}$ contribution. In addition, there is a third-order nonrelativistic perturbative contribution from three one-loop vacuum polarization corrections $[21,26]$ which gives $0.00007 \mathrm{meV}$.

The remaining corrections to the energy levels come from the potentials in Eqs. (1)-(4). In what follows, we use simultaneous eigenstates of $\vec{F}^{2}, F_{z}, \vec{J}^{2}, \vec{S}_{2}^{2}$, where $\vec{F}=\vec{L}+\vec{S}_{1}+\vec{S}_{2}, \vec{J}=\vec{L}+\vec{S}_{1}$

\section{SPIN-INDEPENDENT TERMS}

\section{A. Order $\alpha^{4}$ terms}

The terms in the first line of Eq. (4) contribute to the $s$ and $p$ levels in order $\alpha^{4}$. Their expectation values are (in meV)

$$
\begin{aligned}
\left\langle-\frac{\alpha}{m_{1} m_{2} r} \vec{p}^{2}\right\rangle & =-\frac{\mu^{3} \alpha^{4}}{n^{3} m_{1} m_{2}}\left(\frac{4}{2 \ell+1}-\frac{1}{n}\right) 10^{9}, \\
\left\langle\frac{\alpha \pi}{2 \mu^{2}} \delta(\vec{r})\right\rangle & =\frac{\mu \alpha^{4}}{2 n^{3}} \delta_{\ell 0} 10^{9},
\end{aligned}
$$$$
\left\langle-\frac{1}{8}\left(\frac{1}{m_{1}^{3}}+\frac{1}{m_{2}^{3}}\right)\left(\vec{p}^{2}\right)^{2}\right\rangle
$$$$
=-\frac{\mu \alpha^{4}}{8 n^{3}}\left(\frac{\mu^{3}}{m_{1}^{3}}+\frac{\mu^{3}}{m_{2}^{3}}\right)\left(\frac{8}{2 \ell+1}-\frac{3}{n}\right) 10^{9},
$$

$$
\left\langle\frac{\alpha^{2} \mu}{2 m_{1} m_{2} r^{2}}\right\rangle=\frac{\mu^{3} \alpha^{4}}{n^{3} m_{1} m_{2}} \frac{1}{2 \ell+1} 10^{9},
$$

and it should be noted that the $1 / r^{2}$ term is part of the oneloop correction. For the $n=2$ state, the contributions are

$$
\begin{aligned}
E_{2}(2 S) & =-10.711 \mathrm{meV}, \\
E_{2}(2 P) & =-5.100 \mathrm{meV}, \\
E_{2} & =E_{2}(2 P)-E_{2}(2 S)=5.610 \mathrm{meV} .
\end{aligned}
$$

\section{B. Order $\alpha^{5}$ terms}

The remainder of the terms in Eq. (4) are of order $\alpha^{5}$ or $\alpha^{5} \ln (\alpha)$. There are two issues to address when evaluating 
these terms. The first is the elimination of the photon mass dependence. This is accomplished by using the "Bethe logarithm" technique, which amounts to the replacement of $\ln \left(\mu / \lambda_{I F}\right)$ by [27]

$$
\left(\ln \frac{R_{\infty}}{\alpha^{2} k_{0}(n, 0)}+\frac{5}{6}\right) \delta_{\ell 0}+\ln \frac{R_{\infty}}{k_{0}(n, \ell)}\left(1-\delta_{\ell 0}\right) .
$$

The other is the matrix element of $\nabla^{2}[(\ln (\mu r)+\gamma) / r]$. For states with $\ell>0$, this reduces to $-1 / r^{3}$. When $\ell=0$, the result is

$$
\begin{aligned}
& \left\langle n 0\left|\frac{1}{2 \pi} \nabla^{2}\left[\frac{\ln (\mu r)+\gamma}{r}\right]\right| n 0\right\rangle \\
& \quad=\frac{2 \mu^{3} \alpha^{3}}{\pi n^{3}}\left[\ln \frac{2 \alpha}{n}+\frac{n-1}{2 n}+\sum_{k=1}^{n} \frac{1}{k}\right] .
\end{aligned}
$$

Using

$$
\begin{gathered}
\ln k_{0}(2 S)=2.8117699 \\
\ln k_{0}(2 P)=-0.0300167
\end{gathered}
$$

and denoting the expectation values of the order $\alpha^{5}$ as $E_{4}(n \ell)$, the results are

$$
\begin{aligned}
E_{4}(2 S) & =0.7077 \mathrm{meV}, \\
E_{4}(2 P) & =0.0004 \mathrm{meV}, \\
E_{4} & =E_{4}(2 P)-E_{4}(2 S)=-0.7073 \mathrm{meV} .
\end{aligned}
$$

\section{SPIN-DEPENDENT TERMS}

\section{A. $\ell=0$ hyperfine}

The expectation value of $V_{H F}$ affects only $s$ states and is

$$
\begin{aligned}
E_{H F}\left(n S_{1 / 2}\right)= & \frac{\mu^{3} \alpha^{4}}{n^{3} m_{1} m_{2}}\left[\frac{2}{3}\left(1+a_{\mu}\right)(1+\kappa)\right. \\
& \left.+\frac{\alpha}{\pi} \frac{m_{1} m_{2}}{m_{1}^{2}-m_{2}^{2}} \ln \left(\frac{m_{2}^{2}}{m_{1}^{2}}\right)\right](2 s(s+1)-3),
\end{aligned}
$$

$$
E_{H F}\left({ }^{3} S_{1 / 2}\right)=5.704 \mathrm{meV},
$$

$E_{H F}\left({ }^{1} S_{1 / 2}\right)=-17.113 \mathrm{meV}$,

$\Delta E_{H F}\left(S_{1 / 2}\right)=22.817 \mathrm{meV}$.

\section{B. Spin-orbit and tensor terms}

The largest contribution of $V_{L S}$ is that associated with the $\vec{L} \cdot \vec{S}_{1}$ term. It accounts for the fine structure splitting between the $P_{3 / 2}$ and the $P_{1 / 2}$ states. The contribution is
$E_{F S}\left(P_{j_{1}}\right)=\alpha\left[\frac{\left(1+2 a_{\mu}\right)}{2 m_{1}^{2}}+\frac{\left(1+a_{\mu}\right)}{m_{1} m_{2}}\right]\left\langle\frac{1}{r^{3}}\right\rangle\left\langle\vec{L} \cdot \vec{S}_{1}\right\rangle$.

The expectation value of $r^{-3}$ is

$$
\left\langle\frac{1}{r^{3}}\right\rangle=\frac{2 \mu^{3} \alpha^{3}}{n^{3} \ell(\ell+1)(2 \ell+1)} \rightarrow \frac{\mu^{3} \alpha^{3}}{24} .
$$

Since the eigenstates we are using are eigenstates of $\vec{J}^{2}$,

$$
2 \vec{L} \cdot \vec{S}_{1}=\vec{J}^{2}-\vec{L}^{2}-\vec{S}_{1}^{2}=j(j+1)-2-3 / 4
$$

Then,

$E_{F S}\left(P_{j}\right)=\frac{\mu^{3} \alpha^{4}}{48 m_{1}^{2}}\left[\left(1+2 a_{\mu}\right)+\frac{2 m_{1}}{m_{2}}\left(1+a_{\mu}\right)\right]\left\langle\vec{L} \cdot \vec{S}_{1}\right\rangle$,

and

$E_{F S}\left(P_{3 / 2}\right)=2.782 \mathrm{meV}$

$E_{F S}\left(P_{1 / 2}\right)=-5.564 \mathrm{meV}$,

$$
\Delta E_{F S}=E_{F S}\left(P_{3 / 2}\right)-E_{F S}\left(P_{1 / 2}\right)=8.347 \mathrm{meV} .
$$

The remaining spin-dependent terms are the $\vec{L} \cdot \vec{S}_{2}$ portion of Eq. (2) (call it $V_{L S}^{\prime}$ ) and $V_{T}$. Their matrix elements for a generic $2 P$ state are

$$
\begin{array}{r}
\left\langle 2 P\left|V_{L S}^{\prime}\right| 2 P\right\rangle=\frac{\mu^{3} \alpha^{4}(1+\kappa)}{24 m_{1} m_{2}}\left[1+\frac{m_{1}}{2 m_{2}} \frac{(1+2 \kappa)}{(1+\kappa)}\right]\left\langle\vec{L} \cdot \vec{S}_{2}\right\rangle, \\
\left\langle 2 P\left|V_{T}^{\prime}\right| 2 P\right\rangle=\frac{\mu^{3} \alpha^{4}\left(1+a_{\mu}\right)(1+\kappa)}{24 m_{1} m_{2}}\left\langle 3 \vec{S}_{1} \cdot \hat{r} \vec{S}_{2} \cdot \hat{r}-\vec{S}_{1} \cdot \vec{S}_{2}\right\rangle .
\end{array}
$$

For $V_{L S}^{\prime}$, we can use the fact that $\vec{F}^{2}$ and $\vec{J}^{2}$ are diagonal, so $\vec{F}^{2}=\left(\vec{J}+\vec{S}_{2}\right)^{2}$ and we can obtain the relation

$2 \vec{L} \cdot \vec{S}_{2}=\vec{F}^{2}-\vec{J}^{2}-2 \vec{S}_{1} \cdot \vec{S}_{2}-3 / 4=\vec{F}^{2}-\vec{J}^{2}+3 / 4-\vec{S}^{2}$

Both the ${ }^{5} P_{3 / 2}$ and ${ }^{1} P_{1 / 2}$ states are eigenstates of $\vec{S}^{2}$ with eigenvalue 2 , so $\left\langle{ }^{5} P_{3 / 2}\left|\vec{L} \cdot \vec{S}_{2}\right|^{5} P_{3 / 2}\right\rangle=1 / 2$ and $\left\langle{ }^{1} P_{1 / 2}\left|\vec{L} \cdot \vec{S}_{2}\right|{ }^{1} P_{1 / 2}\right\rangle=-1$ : 


$$
\left\langle{ }^{1} P_{1 / 2}\left|V_{L S}^{\prime}\right|{ }^{1} P_{1 / 2}\right\rangle=-\frac{\mu^{3} \alpha^{4}(1+\kappa)}{24 m_{1} m_{2}}\left[1+\frac{1}{2} \frac{m_{1}}{m_{2}} \frac{(1+2 \kappa)}{(1+\kappa)}\right],
$$

$\left\langle{ }^{5} P_{3 / 2}\left|V_{L S}^{\prime}\right|{ }^{5} P_{3 / 2}\right\rangle=\frac{\mu^{3} \alpha^{4}(1+\kappa)}{48 m_{1} m_{2}}\left[1+\frac{1}{2} \frac{m_{1}}{m_{2}} \frac{(1+2 \kappa)}{(1+\kappa)}\right]$.

The matrix elements of $V_{T}$ for these states are

$$
\begin{aligned}
& \left\langle{ }^{1} P_{1 / 2}\left|V_{T}\right|{ }^{1} P_{1 / 2}\right\rangle=-\frac{\mu^{3} \alpha^{4}(1+\kappa)}{3 m_{1} m_{2}} \frac{\left(1+a_{\mu}\right)}{8}, \\
& \left\langle{ }^{5} P_{3 / 2}\left|V_{T}\right|{ }^{5} P_{3 / 2}\right\rangle=-\frac{\mu^{3} \alpha^{4}(1+\kappa)}{3 m_{1} m_{2}} \frac{\left(1+a_{\mu}\right)}{80} .
\end{aligned}
$$

Combining these two contributions gives

$$
\begin{aligned}
E_{H F S}\left({ }^{1} P_{1 / 2}\right) & =-\frac{\mu^{3} \alpha^{4}(1+\kappa)}{3 m_{1} m_{2}}\left[\frac{1}{4}+\frac{a_{\mu}}{8}+\frac{1}{16} \frac{m_{1}}{m_{2}} \frac{(1+2 \kappa)}{(1+\kappa)}\right] \\
& \rightarrow-5.968 \mathrm{meV}, \\
E_{H F S}\left({ }^{5} P_{3 / 2}\right) & =\frac{\mu^{3} \alpha^{4}(1+\kappa)}{3 m_{1} m_{2}}\left[\frac{1}{20}-\frac{a_{\mu}}{80}+\frac{1}{32} \frac{m_{1}}{m_{2}} \frac{(1+2 \kappa)}{(1+\kappa)}\right] \\
& \rightarrow 1.272 \mathrm{meV} .
\end{aligned}
$$

The ${ }^{3} P_{3 / 2}$ and ${ }^{3} P_{1 / 2}$ states are mixed by the $V_{L S}^{\prime}$ and $V_{T}$ potentials. This results in

$$
\begin{aligned}
& \left\langle{ }^{3} P_{1 / 2}\left|\vec{L} \cdot \vec{S}_{2}\right|{ }^{3} P_{1 / 2}\right\rangle=1 / 3, \\
& \left\langle{ }^{3} P_{3 / 2}\left|\vec{L} \cdot \vec{S}_{2}\right|{ }^{3} P_{3 / 2}\right\rangle=-5 / 6, \\
& \left\langle{ }^{3} P_{3 / 2}\left|\vec{L} \cdot \vec{S}_{2}\right|{ }^{3} P_{1 / 2}\right\rangle=-\sqrt{2} / 3
\end{aligned}
$$

and

$$
\begin{aligned}
& \left\langle{ }^{3} P_{1 / 2}\left|3 \vec{S}_{1} \cdot \hat{r} \vec{S}_{2} \cdot \hat{r}-\vec{S}_{1} \cdot \vec{S}_{2}\right|{ }^{3} P_{1 / 2}\right\rangle=1 / 3, \\
& \left\langle{ }^{3} P_{3 / 2}\left|3 \vec{S}_{1} \cdot \hat{r} \vec{S}_{2} \cdot \hat{r}-\vec{S}_{1} \cdot \vec{S}_{2}\right|{ }^{3} P_{3 / 2}\right\rangle=1 / 6, \\
& \left\langle{ }^{3} P_{3 / 2}\left|3 \vec{S}_{1} \cdot \hat{r} \vec{S}_{2} \cdot \hat{r}-\vec{S}_{1} \cdot \vec{S}_{2}\right|{ }^{3} P_{1 / 2}\right\rangle=\sqrt{2} / 6 .
\end{aligned}
$$

The matrix elements of $V_{L S}^{\prime}$ are

$$
\begin{aligned}
& \left\langle{ }^{3} P_{1 / 2}\left|V_{L S}^{\prime}\right|{ }^{3} P_{1 / 2}\right\rangle=\frac{\mu^{3} \alpha^{4}(1+\kappa)}{72 m_{1} m_{2}}\left[1+\frac{1}{2} \frac{m_{1}}{m_{2}} \frac{(1+2 \kappa)}{(1+\kappa)}\right], \\
& \left\langle{ }^{3} P_{3 / 2}\left|V_{L S}^{\prime}\right|{ }^{3} P_{3 / 2}\right\rangle=-\frac{5 \mu^{3} \alpha^{4}(1+\kappa)}{144 m_{1} m_{2}}\left[1+\frac{1}{2} \frac{m_{1}}{m_{2}} \frac{(1+2 \kappa)}{(1+\kappa)}\right],
\end{aligned}
$$

$$
\left\langle{ }^{3} P_{3 / 2}\left|V_{L S}^{\prime}\right|{ }^{3} P_{1 / 2}\right\rangle=-\frac{\mu^{3} \alpha^{4}(1+\kappa)}{72 m_{1} m_{2}}\left[1+\frac{1}{2} \frac{m_{1}}{m_{2}} \frac{(1+2 \kappa)}{(1+\kappa)}\right] \sqrt{2},
$$

and those of $V_{T}$ are

$$
\left\langle{ }^{3} P_{1 / 2}\left|V_{T}\right|^{3} P_{1 / 2}\right\rangle=\frac{\mu^{3} \alpha^{4}}{72 m_{1} m_{2}}(1+\kappa)\left(1+a_{\mu}\right),
$$

$\left\langle{ }^{3} P_{3 / 2}\left|V_{T}\right|^{3} P_{3 / 2}\right\rangle=\frac{\mu^{3} \alpha^{4}}{144 m_{1} m_{2}}(1+\kappa)\left(1+a_{\mu}\right)$,

$\left\langle{ }^{3} P_{3 / 2}\left|V_{T}\right|^{3} P_{1 / 2}\right\rangle=\frac{\mu^{3} \alpha^{4}}{144 m_{1} m_{2}}(1+\kappa)\left(1+a_{\mu}\right) \sqrt{2}$.

The $V_{L S}^{\prime}$ and $V_{T}$ contributions can be combined to give

$$
\begin{aligned}
E_{H F S}\left({ }^{3} P_{1 / 2}\right) & =\frac{\mu^{3} \alpha^{4}(1+\kappa)}{9 m_{1} m_{2}}\left[\frac{1}{4}+\frac{a_{\mu}}{8}+\frac{1}{16} \frac{m_{1}}{m_{2}} \frac{(1+2 \kappa)}{(1+\kappa)}\right] \\
& \rightarrow 1.989 \mathrm{meV}, \\
E_{H F S}\left({ }^{3} P_{3 / 2}\right) & =-\frac{5 \mu^{3} \alpha^{4}(1+\kappa)}{18 m_{1} m_{2}}\left[\frac{1}{10}-\frac{a_{\mu}}{40}+\frac{1}{16} \frac{m_{1}}{m_{2}} \frac{(1+2 \kappa)}{(1+\kappa)}\right] \\
& \rightarrow-2.120 \mathrm{meV},
\end{aligned}
$$

$$
\begin{aligned}
E_{M I X}\left({ }^{3} P_{1 / 2}-{ }^{3} P_{3 / 2}\right) & =-\frac{\mu^{3} \alpha^{4}(1+\kappa)}{3 m_{1} m_{2}}\left[1+\frac{m_{1}}{m_{2}} \frac{(1+2 \kappa)}{(1+\kappa)}\right] \frac{\sqrt{2}}{48} \\
& \rightarrow-0.796 \mathrm{meV} .
\end{aligned}
$$

The expression for $E_{M I X}$ omits the $a_{\mu}$ contribution and all these results agree with Ref. [5].

Diagonalizing the triplet $P$ mixing matrix

$$
\left(\begin{array}{cc}
E_{H F S}\left({ }^{3} P_{1 / 2}\right) & E_{M I X} \\
E_{M I X} & E_{H F S}\left({ }^{3} P_{3 / 2}\right)+\Delta E_{F S}
\end{array}\right)
$$

has the effect of shifting the ${ }^{3} P_{3 / 2}$ level up by $\Delta=$ $0.1447 \mathrm{meV}$ and the ${ }^{3} P_{1 / 2}$ level down by the same amount.

There are small electron vacuum polarization corrections to all of the terms in the potential that contribute to order $\alpha^{4}$. These are computed in Appendix A and included in the results that are compared with the experiment.

\section{RESULTS AND CONCLUSIONS}

Relative to the $n=2$ Bohr level, the energies of the various $n=2$ states, including the small corrections calculated in Appendix A, are 


$$
\begin{aligned}
& E\left({ }^{5} P_{3 / 2}\right)= E E_{V P}+E_{2}+E_{4}+E_{F S}\left(P_{3 / 2}\right)+E_{H F S}\left({ }^{5} P_{3 / 2}\right) \\
&= 215.609 \mathrm{meV}, \\
& E\left({ }^{3} P_{3 / 2}\right)= E E_{V P}+E_{2}+E_{4}+E_{F S}\left(P_{3 / 2}\right)+E_{H F S}\left({ }^{3} P_{3 / 2}\right) \\
&+\Delta=212.360 \mathrm{meV}, \\
& E\left({ }^{3} P_{1 / 2}\right)= E E_{V P}+E_{2}+E_{4}+E_{F S}\left(P_{1 / 2}\right) \\
&+E_{H F S}\left({ }^{3} P_{1 / 2}\right)-\Delta=207.826 \mathrm{meV}, \\
& E\left({ }^{1} P_{1 / 2}\right)= E E_{V P}+E_{2}+E_{4}+E_{F S}\left(P_{1 / 2}\right) \\
&+E_{H F S}\left({ }^{1} P_{1 / 2}\right)=200.006 \mathrm{meV}, \\
& E\left({ }^{3} S_{1 / 2}\right)= E_{H F}\left({ }^{3} S_{1 / 2}\right)=5.7351 \mathrm{meV}, \\
& E\left({ }^{1} S_{1 / 2}\right)= E_{H F}\left({ }^{1} S_{1 / 2}\right)=-17.2054 \mathrm{meV}, \\
& E\left(P_{1 / 2}\right)- E\left(S_{1 / 2}\right)=E E_{V P}+E_{2}+E_{4}+E_{F S}\left(P_{1 / 2}\right) \\
& \quad=205.980 \mathrm{meV} .
\end{aligned}
$$

Here, $E E_{V P}$ is the sum of the first seven rows in Table I, $E_{2}$ is the eighth row, $E_{4}$ the ninth row, $E_{F S}$ the tenth row, the $s$-state hyperfine splitting the eleventh row and the $p$-state hyperfine splittings the twelfth row. The additional small corrections in Appendix A include the spinindependent terms in subsection $\mathrm{I}$, the $\ell=0$ hyperfine splitting in subsection IV, the fine structure splittings in subsection 2 , and the $\ell=1$ hyperfine splitting in subsections 3, 4 and 5. Equation (54g) gives our value for the Lamb shift excluding proton structure corrections.

We have used nonrelativistic wave functions throughout because our potential contains the leading-order relativistic, recoil and one-loop corrections. However, if we use the solutions to the Dirac equation given in Rose [28], the value of the electron one-loop vacuum polarization changes from 205.007 to $205.028 \mathrm{meV}$ for the $2 P_{1 / 2}-2 S_{1 / 2}$ interval and from 205.007 to $205.033 \mathrm{meV}$ for the $2 P_{3 / 2}-2 S_{1 / 2}$ interval [4]. We have also calculated the relativistic corrections to the two-loop vacuum polarization contribution using the approach of [21]. In this order, the change in the $2 P_{1 / 2}-2 S_{1 / 2}$ interval is $0.0001 \mathrm{meV}$ and the corresponding change in the $2 P_{3 / 2}-2 S_{1 / 2}$ interval is $0.0002 \mathrm{meV}$.

A more fundamental way to calculate the relativistic corrections to the dominant electron vacuum polarization contribution would be to use the solutions to the Salpeter equation with an instantaneous Coulomb kernel. Estimates of this correction using the scalar Salpeter equation have been made [29] and the results are small. Unfortunately, analytic solutions for the spin $1 / 2$ Salpeter wave functions

\begin{tabular}{|c|c|c|c|c|c|c|c|}
\hline & ${ }^{1} S_{1 / 2}$ & ${ }^{3} S_{1 / 2}$ & ${ }^{1} P_{1 / 2}$ & ${ }^{3} P_{1 / 2}$ & ${ }^{3} P_{3 / 2}$ & ${ }^{5} P_{3 / 2}$ & ${ }^{3} P_{1 / 2} \leftrightarrow{ }^{3} P_{3 / 2}$ \\
\hline One-loop vacuum polarization & & & 205.007 & 205.007 & 205.007 & 205.007 & \\
\hline Relativistic one-loop correction & & & 0.021 & 0.021 & 0.026 & 0.026 & \\
\hline Two-loop vacuum polarization & & & 1.508 & 1.508 & 1.508 & 1.508 & \\
\hline Relativistic two-loop correction & & & 0.0001 & 0.0001 & 0.0002 & 0.0002 & \\
\hline Three-loop vacuum polarization & & & 0.0044 & 0.0044 & 0.0044 & 0.0044 & \\
\hline NR 2nd-order $\alpha^{4}$ vacuum polarization & & & 0.1509 & 0.1509 & 0.1509 & 0.1509 & \\
\hline NR 2nd-order $\alpha^{5}$ vacuum polarization & & & 0.0022 & 0.0022 & 0.0022 & 0.0022 & \\
\hline Spin-independent $\alpha^{4}$ & & & 5.6102 & 5.6102 & 5.6102 & 5.6102 & \\
\hline Spin-independent $\alpha^{5}$ & & & -0.7073 & -0.7073 & -0.7073 & -0.7073 & \\
\hline Fine structure & & & -5.5645 & -5.5645 & 2.7823 & 2.7823 & \\
\hline Hyperfine $\ell=0$ & -17.113 & 5.7045 & & & & & \\
\hline Hyperfine $\ell=1$ & & & -5.9682 & 1.9894 & -2.1195 & 1.2717 & \\
\hline \multirow[t]{3}{*}{ Mixing matrix element } & & & & & & & -0.79615 \\
\hline & & & Vacuum & olarization & rrections to & $V_{2}(\vec{k})$ & \\
\hline & -0.0920 & 0.0307 & -0.0532 & -0.0464 & -0.0446 & -0.0418 & -0.0003 \\
\hline Total & -17.205 & 5.735 & 200.006 & 207.971 & 212.215 & 215.609 & -0.7965 \\
\hline Result of mixing & & & & 207.826 & 212.360 & & \\
\hline
\end{tabular}
with unequal masses are not available.

Finally, one might wonder how the relatively large contributions from the mass dependence of the delta function term and the one-loop $\alpha^{2} / r^{2}$ term mentioned in the introduction can still lead to a Lamb shift value that is in agreement with other calculations. The answer is that there are two versions of the spin-independent fine structure Hamiltonian that contribute order $\alpha^{4}$ corrections to the $2 P_{1 / 2}-2 S_{1 / 2}$ energy difference. One is the Breit-Pauli version [7] $H_{B-P}^{\prime}$ given by

TABLE I. The entries summarize the various corrections (in meV) to the $n=2$ states of muonic hydrogen calculated using Eqs. (1)-(4). 


$$
\begin{aligned}
H_{B-P}^{\prime} & =\frac{\pi \alpha}{2}\left(\frac{1}{m_{1}^{2}}+\frac{1}{m_{2}^{2}}\right) \delta(\vec{r})-\frac{\alpha}{2 m_{1} m_{2}} p_{i}\left(\frac{\delta_{i j}}{r}+\frac{x_{i} x_{j}}{r^{3}}\right) p_{j} \\
& =\frac{\pi \alpha}{2 \mu^{2}} \delta(\vec{r})-\frac{\pi \alpha}{m_{1} m_{2}} \delta(\vec{r})-\frac{\alpha}{2 m_{1} m_{2}} p_{i}\left(\frac{\delta_{i j}}{r}+\frac{x_{i} x_{j}}{r^{3}}\right) p_{j}
\end{aligned}
$$

The other is $H_{G R S}^{\prime}$ of Ref. [14], which has the form

$$
H_{G R S}^{\prime}=\frac{\pi \alpha}{2 \mu^{2}} \delta(\vec{r})-\frac{\alpha}{m_{1} m_{2}} \frac{1}{r} \vec{p}^{2}+\frac{\mu \alpha^{2}}{2 m_{1} m_{2} r^{2}},
$$

where the last term arises in the calculation of the one-loop corrections. The last two terms of Eqs. (56) and (57) give identical contributions to the $2 P_{1 / 2}-2 S_{1 / 2}$ splitting, namely,

$$
\Delta E(2 P-2 S)=\frac{\mu^{3} \alpha^{4}}{4 m_{1} m_{2}}
$$

All the spin-dependent fine structure terms of the two versions are the same so, apart from minor differences in some recoil terms, the Lamb shift values agree. This implies that there should be no $\alpha^{2} / r^{2}$ term associated with the one-loop corrections to Breit-Pauli Hamiltonian. In Appendix B this is shown to be the case.

\section{ACKNOWLEDGMENTS}

W. W. R. would like to thank Levere Hostler, who introduced him to the properties of the Coulomb Green's function and Stanley Radford for a discussion about effective potentials.

\section{APPENDIX A: ELECTRON VACUUM POLARIZATION CORRECTIONS TO ORDER $\alpha^{4}$ TERMS}

The electron vacuum polarization corrections to the leading-order $\alpha^{4}$ terms in the potential can be obtained from the momentum space representation of the potential, which is

$$
\begin{aligned}
V_{2}(\vec{k})= & -\frac{e^{2}}{\vec{k}^{2}}\left[1-\frac{\vec{k}^{2}}{8 \mu^{2}}+\frac{i}{4 m_{1} m_{2}}\left(\left(2+\frac{m_{2}}{m_{1}}\right)(\vec{k} \times \vec{p}) \cdot \vec{\sigma}_{1}+(1+\kappa)\left(2+\frac{1+2 \kappa}{1+\kappa} \frac{m_{1}}{m_{2}}\right)(\vec{k} \times \vec{p}) \cdot \vec{\sigma}_{2}\right)\right. \\
& \left.-\frac{(1+\kappa)}{6 m_{1} m_{2}} \vec{k}^{2} \vec{\sigma}_{1} \cdot \vec{\sigma}_{2}+\frac{(1+\kappa)}{4 m_{1} m_{2}}\left(\vec{k} \cdot \vec{\sigma}_{1} \vec{k} \cdot \vec{\sigma}_{2}-\frac{1}{3} \vec{k}^{2} \vec{\sigma}_{1} \cdot \vec{\sigma}_{2}\right)+\frac{\vec{p}^{2}}{m_{1} m_{2}}-\frac{\alpha \mu \pi k}{4 m_{1} m_{2}}-\frac{1}{6}\left\langle r^{2}\right\rangle \vec{k}^{2}\right],
\end{aligned}
$$

where the next to last term is a one-loop correction that contributes at order $\alpha^{4}$. The electron vacuum polarization correction is obtained by making the replacement

$$
-\frac{e^{2}}{\vec{k}^{2}} \rightarrow-e^{2} \int_{4 m_{e}^{2}}^{\infty} \frac{d \lambda}{\lambda} \frac{\Delta^{(2)}(\lambda)}{\vec{k}^{2}+\lambda}
$$

In addition, there is a second-order perturbative correction containing $V_{V P}(r)$ for each of these terms as well as for the relativistic kinetic energy terms. In these calculations, all integrals except those over $\lambda$ can be evaluated analytically using Mathematica. The integrals over $\lambda$ are performed using the Mathematica NIntegrate routine.

\section{Spin-independent terms}

$$
\text { a. } e^{2} \vec{k}^{2} / 8 \mu^{2}
$$

This leads to the expression

$$
\frac{\pi \alpha}{2 \mu^{2}} \int_{4 m_{e}^{2}}^{\infty} \frac{d \lambda \Pi_{e}(\lambda)}{\lambda} \frac{\vec{k}^{2}}{\vec{k}^{2}+\lambda},
$$

and transforming to coordinate space gives

$$
\frac{\pi \alpha}{2 \mu^{2}} \int_{4 m_{e}^{2}}^{\infty} \frac{d \lambda \Pi_{e}(\lambda)}{\lambda}\left(\delta(\vec{r})-\frac{\lambda}{4 \pi} \frac{1}{r} e^{-\sqrt{\lambda} r}\right) .
$$

The delta function only contributes to $\ell=0$ and gives

$$
\Delta E(\delta)=\frac{\mu \alpha^{5}}{48 \pi} \int_{4}^{\infty} \frac{d x}{x}(1+2 / x) \sqrt{1-4 / x} .
$$

The integral diverges, but the $\ell=0$ contribution of the remaining term cancels this divergence. Using Eq. (14) and the extra factor of $\lambda=m_{e}^{2} x$, the remaining contribution to the $E(2 P)-E(2 S)$ interval is

$$
\begin{aligned}
\Delta( & E(2 P)-E(2 S)) \\
& =\frac{\mu \alpha^{5}}{48 \pi} \int_{4}^{\infty} \frac{d x m_{e}^{4} a^{4} x^{2}(1+2 / x) \sqrt{1-4 / x}}{x\left(1+m_{e} a \sqrt{x}\right)^{4}} .
\end{aligned}
$$

The vacuum polarization correction to this term is

$$
\begin{aligned}
\Delta E_{1}\left(e^{2} \vec{k}^{2} / 8 \mu^{2}\right)= & \frac{\mu \alpha^{5}}{48 \pi} \int_{4}^{\infty} \frac{d x}{x}(1+2 / x) \sqrt{1-4 / x} \\
& \times\left[\frac{m_{e}^{4} a^{4} x^{2}}{\left(1+m_{e} a \sqrt{x}\right)^{4}}-1\right] \\
= & -0.0363 \mathrm{meV} .
\end{aligned}
$$


The second-order correction is

$$
\begin{aligned}
\Delta E_{2}\left(e^{2} \delta(\vec{r}) / 8 \mu^{2}\right) & =2\left(\frac{\pi \alpha}{2 \mu^{2}}\right) \int d^{3} r \psi_{20}(\vec{r}) V_{V P}(r) \int d r^{\prime 3} g_{20}\left(r, r^{\prime}\right) \delta(\vec{r}) \psi_{20}(\vec{r}) \\
& =-\left(\frac{\pi \alpha^{2}}{\mu^{2}}\right) \int d^{3} r \frac{R_{20}(r)}{8 \pi a^{3}} \frac{1}{r} e^{-\sqrt{\lambda} r} g_{20}(r / a, 0) \\
& =-\left(\frac{\pi \alpha^{2}}{2 \mu^{2} a^{3}}\right) \int_{0}^{\infty} d r r\left(1-\frac{r}{2 a}\right) e^{-r / 2 a} e^{-\sqrt{\lambda} r} g_{20}(r / a, 0), \\
\Delta E_{2}\left(e^{2} \delta(\vec{r}) / 8 \mu^{2}\right) & =-\left(\frac{\pi \alpha^{3}}{2 \mu}\right) \int_{0}^{\infty} d x x(1-x / 2) e^{-x / 2} e^{-\sqrt{\lambda} a x} g_{20}(x, 0),
\end{aligned}
$$

where the integration over $d \lambda$ has been suppressed. The Greens function $g_{20}(x, 0)$ is

$$
g_{20}(x, 0)=\frac{\mu^{2} \alpha}{4 \pi} \frac{e^{-x / 2}}{2 x}\left[-4+(8 \gamma-6) x+(13-4 \gamma) x^{2}-x^{3}-4 x(x-2) \ln (x)\right]=\frac{\mu^{2} \alpha}{4 \pi} \mathcal{G}_{20}(x) .
$$

The final expression for the $2 p-2 s$ splitting is

$$
-\Delta E_{2}\left(e^{2} \delta(\vec{r}) / 8 \mu^{2}\right)=\frac{\mu \alpha^{4}}{8} \frac{\alpha}{3 \pi} \int_{4}^{\infty} \frac{d x}{x}(1+2 / x) \sqrt{1-4 / x} F(\beta \sqrt{x})=-0.0549 \mathrm{meV},
$$

where

$$
\begin{aligned}
& F(\beta \sqrt{x})=\int_{0}^{\infty} d y y(1-y / 2) e^{-y / 2} e^{-\beta \sqrt{x} y} \mathcal{G}_{20}(y) \\
& F(\beta \sqrt{x})=-\frac{3+11 \beta \sqrt{x}+4 \beta^{2} x+12 \beta^{3} x^{3 / 2}+4 \beta^{4} x^{2}+4(1+\beta \sqrt{x})\left(1+2 \beta^{2} x\right) \ln (1+\beta \sqrt{x})}{2(1+\beta \sqrt{x})^{5}},
\end{aligned}
$$

and $\beta=m_{e} a$.

Here, the expression is

$$
\text { b. }-e^{2} \vec{p}^{2} / r m_{1} m_{2}
$$

$$
\frac{-e^{2}}{m_{1} m_{2}} \frac{1}{(2 \pi)^{3}} \int \frac{d^{3} k e^{i \vec{k} \cdot \vec{r}}}{\vec{k}^{2}+\lambda} \vec{p}^{2}=-\frac{\alpha}{m_{1} m_{2} r} e^{-\sqrt{\lambda} r} \vec{p}^{2}=-\frac{2 \mu \alpha^{2}}{m_{1} m_{2} r^{2}}\left(1-\frac{r}{8 a}\right) e^{-\sqrt{\lambda} r}
$$

For the $\ell=1$ state, the result of integrating over $r$ is

$$
\frac{\mu^{3} \alpha^{4}}{12 m_{1} m_{2}} \frac{1}{(1+a \sqrt{\lambda})^{4}}\left(\frac{3}{4}-2(1+a \sqrt{\lambda})\right) .
$$

The $\ell=0$ state when integrated over $r$ gives

$$
\frac{\mu^{3} \alpha^{4}}{m_{1} m_{2}} \frac{1}{(1+a \sqrt{\lambda})^{4}}\left(-(1+a \sqrt{\lambda})^{3}+\frac{9}{8}(1+a \sqrt{\lambda})^{2}-\frac{3}{4}(1+a \sqrt{\lambda})+\frac{3}{16}\right) .
$$

Integrating the difference of these two results over $\lambda$ gives

$$
\Delta E_{1}\left(-e^{2} \vec{p}^{2} / r m_{1} m_{2}\right)=0.0142 \mathrm{meV} .
$$

The expression for the second-order correction is (again, suppressing the integral over $d \lambda$ and including the factor of 2)

$$
\Delta E_{2}\left(-e^{2} \vec{p}^{2} / r m_{1} m_{2}\right)=\frac{4 \mu^{3} \alpha^{4}}{m_{1} m_{2}} \int_{0}^{\infty} d r r R_{2 \ell}(r) e^{-\sqrt{\lambda} r} \int_{0}^{\infty} d r^{\prime} g_{2 \ell}\left(r / a, r^{\prime} / a\right)\left(1-r^{\prime} / 8 a\right) R_{2 \ell}\left(r^{\prime}\right) .
$$

This expression reduces to 


$$
\frac{\mu^{3} \alpha^{4}}{6 m_{1} m_{2}} \int_{0}^{\infty} d x x^{2} e^{-x / 2} e^{-\sqrt{\lambda} a x} \int_{0}^{\infty} d x^{\prime} g_{21}\left(x, x^{\prime}\right) x^{\prime} e^{-x^{\prime} / 2}\left(1-x^{\prime} / 8\right)
$$

for $\ell=1$ and

$$
\frac{2 \mu^{3} \alpha^{4}}{m_{1} m_{2}} \int_{0}^{\infty} d x x(1-x / 2) e^{-x / 2} e^{-\sqrt{\lambda} a x} \int_{0}^{\infty} d x^{\prime} g_{20}\left(x, x^{\prime}\right)\left(1-x^{\prime} / 2\right) e^{-x^{\prime} / 2}\left(1-x^{\prime} / 8\right)
$$

for $\ell=0$. The integrals are, respectively,

$$
-\frac{13+33 a \sqrt{\lambda}+32(1+a \sqrt{\lambda}) \ln (1+a \sqrt{\lambda})}{8(1+a \sqrt{\lambda})^{5}}
$$

and

$$
-\frac{30+86 a \sqrt{\lambda}-60 a^{2} \lambda+52 a^{3} \lambda^{3 / 2}+64(1+a \sqrt{\lambda})\left(1+2 a^{2} \lambda\right) \ln (1+a \sqrt{\lambda})}{64(1+a \sqrt{\lambda})^{5}} .
$$

Integrating the difference over $d \lambda$ gives a $2 p-2 s$ contribution of

$$
\Delta E_{2}\left(-e^{2} \vec{p}^{2} / r m_{1} m_{2}\right)=0.01459 \mathrm{meV} .
$$

$$
\text { c. }-\left(\vec{p}^{2}\right)^{2} / 8 m_{1}^{3}
$$

When integrated over $d \lambda$, the second-order correction to the relativistic kinetic energy contribution is

$$
\Delta E_{2}\left(-\left(\vec{p}^{2}\right)^{2} / 8 m_{1}^{3}\right)=\frac{4 \mu^{4} \alpha^{4}}{8 m_{1}^{3}} \int_{0}^{\infty} d r r R_{2 \ell}(r) e^{-\sqrt{\lambda} r} \int_{0}^{\infty} d r^{\prime} g_{2 \ell}\left(r / a, r^{\prime} / a\right)\left(1-r^{\prime} / 8 a\right)^{2} R_{2 \ell}\left(r^{\prime}\right) .
$$

This correction is negligible for $\ell=1$. After completion of the integrals above, the contribution from the muon to the $2 p-2 s$ splitting for $\ell=0$ is

$$
\Delta E_{2}\left(-\left(\vec{p}^{2}\right)^{2} / 8 m_{1}^{3}\right)=\frac{\mu^{4} \alpha^{4}}{2 m_{1}^{3}} \frac{\alpha}{3 \pi} \int_{4}^{\infty} \frac{d x}{x}(1+2 / x) \sqrt{1-4 / x} G(\beta \sqrt{x})=0.02237 \mathrm{meV},
$$

with

$$
G(\beta \sqrt{x})=-\frac{7+\beta \sqrt{x}(19+2 \beta \sqrt{x}(-7+5 \beta \sqrt{x}))+16(1+\beta \sqrt{x})\left(1+2 \beta^{2} x\right) \ln (1+\beta \sqrt{x})}{16(1+\beta \sqrt{x})^{5}} .
$$

\section{d. $\alpha \mu \pi k / 4 m_{1} m_{2}$}

Here, the momentum space integral is

$$
\frac{\mu \alpha^{2} \pi^{2}}{m_{1} m_{2}} \frac{1}{(2 \pi)^{3}} \int \frac{d^{3} k k e^{i \vec{k} \cdot \vec{r}} e^{-\varepsilon k}}{\vec{k}^{2}+\lambda}=\frac{\mu \alpha^{2}}{2 m_{1} m_{2} r^{2}}\left(1-\sqrt{\lambda} r \int_{0}^{\infty} \frac{d t}{t^{2}+1} \sin (\sqrt{\lambda} t r)\right)
$$

where $\varepsilon$ is taken to 0 after the integral is evaluated. The $d t$ integral can be evaluated at this point, but it is more convenient to first integrate over $d r$ with the integrand multiplied by $R_{2 \ell}^{2}(r)$. For the $\ell=1$, the result is

$$
\frac{1}{24 a^{3}} \int_{0}^{\infty} d r\left(1-\sqrt{\lambda} \int_{0}^{\infty} \frac{d t}{t^{2}+1} r \sin (\sqrt{\lambda} t r)\right) \frac{r^{2}}{a^{2}} e^{-r / a}=\frac{1}{24 a^{3}}\left[2+\int_{0}^{\infty} \frac{d t}{t^{2}+1} \frac{24 \lambda a^{2} t\left(\lambda a^{2} t^{2}-1\right)}{\left(1+\lambda a^{2} t^{2}\right)^{4}}\right] .
$$

The integration over $d t$ then gives

$$
\frac{1}{12 a^{2}}\left[1-\frac{\lambda a^{2}\left(\lambda^{3} a^{6}-3 \lambda^{2} a^{4}+15 \lambda a^{2}-6\left(\lambda a^{2}+1\right) \ln \left(\lambda a^{2}\right)-13\right)}{\left(1-\lambda a^{2}\right)^{4}}\right] .
$$

The calculation for $\ell=0$ is similar. Taking the difference and integrating over $\lambda$ results in the expression 


$$
\begin{aligned}
\Delta E_{1}\left(e^{2} \alpha \mu \pi k / 4 m_{1} m_{2}\right)= & \frac{\mu^{3} \alpha^{5}}{72 \pi m_{1} m_{2}} \int_{4}^{\infty} \frac{d x}{x} \frac{(1+2 / x) \sqrt{1-4 / x}}{\left(1-\beta^{2} x\right)^{4}}\left[26 \beta^{6} x^{3}-30 \beta^{4} x^{2}+6 \beta^{2} x\right. \\
& \left.-24 \beta^{4} x^{2}\left(\beta^{2} x+1\right) \ln (\beta \sqrt{x})-2\right]=-0.0030 \mathrm{meV},
\end{aligned}
$$

where $\beta=m_{e} a$.

The second-order correction for $\mu \alpha^{2} / 2 m_{1} m_{2} r^{2}$ is obtained by integrating

$$
\Delta E_{2}(\ell)=-\frac{\mu^{3} \alpha^{4} a^{3}}{2 m_{1} m_{2}} \int_{0}^{\infty} d x x R_{2 \ell}(x) e^{-\sqrt{\lambda} a x} \int_{0}^{\infty} d x^{\prime} g_{2 \ell}\left(x, x^{\prime}\right) R_{2 \ell}\left(x^{\prime}\right)
$$

over $\lambda$. The results are

$$
\Delta E_{2}(P)=0.00003 \mathrm{meV}, \quad \Delta E_{2}(S)=0.00154 \mathrm{meV},
$$

for a $2 p-2 s$ splitting contribution of

$$
\Delta E_{2}\left(e^{2} \alpha \mu \pi k / 4 m_{1} m_{2}\right)=-0.0015 \mathrm{meV} .
$$

\section{Fine structure}

a. $-e^{2} i k_{i} / 4 m_{1} m_{2}$

The integral to be evaluated is

$$
\frac{1}{(2 \pi)^{3}} \int \frac{d k^{3} k_{i} e^{i \vec{k} \cdot \vec{r}}}{\vec{k}^{2}+\lambda}=i \frac{x_{i}}{4 \pi r^{3}}(1+\sqrt{\lambda} r) e^{-\sqrt{\lambda} r} .
$$

This leads to the spin-orbit contributions

$$
\frac{\alpha}{2 m_{1} m_{2} r^{3}}\left[\left(2+\frac{m_{2}}{m_{1}}\right) \vec{L} \cdot \vec{S}_{1}+(1+\kappa)\left(2+\frac{(1+2 \kappa) m_{1}}{(1+\kappa) m_{2}}\right) \vec{L} \cdot \vec{S}_{2}\right](1+\sqrt{\lambda} r) e^{-\sqrt{\lambda} r}
$$

Only the $p$ state is affected and we have

$$
\left\langle 2 p\left|\frac{1}{r^{3}}(1+\sqrt{\lambda} r) e^{-\sqrt{\lambda} r}\right| 2 p\right\rangle=\frac{1}{24 a^{3}} \frac{1+3 a \sqrt{\lambda}}{(1+a \sqrt{\lambda})^{3}} .
$$

For the $\vec{L} \cdot \vec{S}_{1}$ term, $\left\langle\vec{L} \cdot \vec{S}_{1}\right\rangle$ is $1 / 2$ for the $P_{3 / 2}$ states and -1 for the $P_{1 / 2}$ states. Thus, their (fine structure) contributions are

$$
\frac{\mu^{3} \alpha^{5}}{144 \pi m_{1}^{2}}\left(1+2 m_{1} / m_{2}\right)\left\langle\vec{L} \cdot \vec{S}_{1}\right\rangle \int_{4}^{\infty} \frac{d x}{x} \frac{(1+2 / x) \sqrt{1-4 / x}(1+3 \beta \sqrt{x})}{(1+\beta \sqrt{x})^{3}}=\left\{\begin{array}{l}
\Delta E_{1 F S}\left(P_{3 / 2}\right)=0.001 \mathrm{meV} \\
\Delta E_{1 F S}\left(P_{1 / 2}\right)=-0.002 \mathrm{meV}
\end{array}\right.
$$

The second-order contribution can be obtained from the expression

$$
\Delta E_{2 F S}(P)=-\frac{\mu^{3} \alpha^{4}}{24 m_{1}^{2}}\left(1+2 m_{1} / m_{2}\right)\left\langle\vec{L} \cdot \vec{S}_{1}\right\rangle \int_{0}^{\infty} d t t^{2} e^{-t / 2} e^{-\sqrt{\lambda} a t} \int_{0}^{\infty} d t^{\prime} g_{21}\left(t, t^{\prime}\right) e^{-t^{\prime} / 2},
$$

integrated over $\lambda$. The result is

$$
-\frac{\mu^{3} \alpha^{5}}{72 m_{1}^{2}}\left(1+2 m_{1} / m_{2}\right)\left\langle\vec{L} \cdot \vec{S}_{1}\right\rangle \int_{4}^{\infty} \frac{d x}{x}(1+2 / x) \sqrt{1-4 / x} H(\beta)=\left\{\begin{array}{l}
\Delta E_{2 F S}\left(P_{3 / 2}\right)=0.0006 \mathrm{meV}, \\
\Delta E_{2 F S}\left(P_{1 / 2}\right)=-0.0012 \mathrm{meV},
\end{array}\right.
$$

with

$$
H(\beta \sqrt{x})=-\frac{3+\beta \sqrt{x}(11+4 \beta \sqrt{x})+4(1+\beta \sqrt{x}) \ln (1+\beta \sqrt{x})}{2(1+\beta \sqrt{x})^{5}}
$$




\section{3. $p$ state hyperfine splitting}

The $\vec{L} \cdot \vec{S}_{2}$ term gives corrections to the hyperfine splitting. The values of these corrections are obtained from Eq. (A35) by changing the coefficient of the integral to

$$
\frac{\mu^{3} \alpha^{5}}{144 \pi m_{1} m_{2}}(1+\kappa)\left(2+\frac{(1+2 \kappa)}{(1+\kappa)} \frac{m_{1}}{m_{2}}\right)\left\langle\vec{L} \cdot \vec{S}_{2}\right\rangle
$$

and using

$$
\begin{aligned}
& \left\langle{ }^{5} P_{3 / 2}\left|\vec{L} \cdot \vec{S}_{2}\right|{ }^{5} P_{3 / 2}\right\rangle=\frac{1}{2}, \\
& \left\langle{ }^{3} P_{3 / 2}\left|\vec{L} \cdot \vec{S}_{2}\right|{ }^{3} P_{3 / 2}\right\rangle=-\frac{5}{6}, \\
& \left\langle{ }^{3} P_{1 / 2}\left|\vec{L} \cdot \vec{S}_{2}\right|{ }^{3} P_{1 / 2}\right\rangle=\frac{1}{3}, \\
& \left\langle{ }^{1} P_{1 / 2}\left|\vec{L} \cdot \vec{S}_{2}\right|{ }^{1} P_{1 / 2}\right\rangle=-1, \\
& \left\langle{ }^{3} P_{3 / 2}\left|\vec{L} \cdot \vec{S}_{2}\right|{ }^{3} P_{1 / 2}\right\rangle=-\sqrt{2} / 3 .
\end{aligned}
$$

The corresponding energy corrections are

$$
\begin{aligned}
& \Delta E_{1 H F}\left({ }^{5} P_{3 / 2}\right)=0.0006 \mathrm{meV}, \\
& \Delta E_{1 H F}\left({ }^{3} P_{3 / 2}\right)=-0.0009 \mathrm{meV}, \\
& \Delta E_{1 H F}\left({ }^{3} P_{1 / 2}\right)=0.0004 \mathrm{meV}, \\
& \Delta E_{1 H F}\left({ }^{1} P_{1 / 2}\right)=-0.0011 \mathrm{meV}, \\
& \Delta E_{1 H F}(M i x)=-0.0005 \mathrm{meV} .
\end{aligned}
$$

The second-order perturbative hyperfine corrections can be obtained from Eq. (A37) by replacing its coefficient with

$$
-\frac{\mu^{3} \alpha^{5}(1+\kappa)}{72 m_{1} m_{2}}\left(2+\frac{(1+2 \kappa)}{(1+\kappa)}\right)\left\langle\vec{L} \cdot \vec{S}_{2}\right\rangle .
$$

The results are

$$
\begin{aligned}
& \Delta E_{2 H F}\left({ }^{5} P_{3 / 2}\right)=0.0004 \mathrm{meV}, \\
& \Delta E_{2 H F}\left({ }^{3} P_{3 / 2}\right)=-0.0006 \mathrm{meV}, \\
& \Delta E_{2 H F}\left({ }^{3} P_{1 / 2}\right)=0.0002 \mathrm{meV}, \\
& \Delta E_{2 H F}\left({ }^{1} P_{1 / 2}\right)=-0.0007 \mathrm{meV}, \\
& \Delta E_{2 H F}(M i x)=-0.0003 \mathrm{meV} .
\end{aligned}
$$

\section{Hyperfine splitting}

$$
\text { a. } e^{2}(1+\kappa) \vec{k}^{2} \sigma_{1} \cdot \sigma_{2} / 6 m_{1} m_{2}
$$

This term is very similar to the spin-independent $\vec{k}^{2}$ contribution treated above. The spin dependence means that the $s$ states and $p$ states must be treated separately. For the $s$ state, we have

$$
\Delta V S_{H F}(r)=\frac{\alpha(1+\kappa)}{6 m_{1} m_{2}} \int_{4 m_{e}^{2}}^{\infty} \frac{d \lambda}{\lambda} \Pi_{e}(\lambda)\left[4 \pi \delta(\vec{r})-\frac{\lambda}{r} e^{-\sqrt{\lambda} r}\right] \sigma_{1} \cdot \sigma_{2} .
$$

The delta function gives

$$
\frac{\mu^{3} \alpha^{4}(1+\kappa)}{12 m_{1} m_{2}} \sigma_{1} \cdot \sigma_{2} \int_{4 m_{e}^{2}}^{\infty} \frac{d \lambda}{\lambda} \Pi_{e}(\lambda)
$$

and the remaining term gives

$$
-\frac{\mu^{3} \alpha^{4}(1+\kappa)}{12 m_{1} m_{2}} \sigma_{1} \cdot \sigma_{2} \int_{4 m_{e}^{2}}^{\infty} \frac{d \lambda}{\lambda} \Pi_{e}(\lambda)\left[\frac{a^{4} \lambda^{2}+a^{2} \lambda / 2}{(1+a \sqrt{\lambda})^{4}}\right] .
$$

The energy corrections are

$$
\begin{aligned}
\Delta E_{1 H F}(S) & =\frac{\mu^{3} \alpha^{5}(1+\kappa)}{9 \pi m_{1} m_{2}}\left\langle\vec{S}_{1} \cdot \vec{S}_{2}\right\rangle \int_{4}^{\infty} \frac{d x}{x} \frac{(1+2 / x) \sqrt{1-4 / x}\left(1+4 \beta \sqrt{x}+11 \beta^{2} x / 2+4 \beta^{3} x^{3 / 2}\right)}{(1+\beta \sqrt{x})^{4}} \\
& =\left\{\begin{array}{l}
\Delta E_{1 H F}\left({ }^{3} S_{1 / 2}\right)=0.0121 \mathrm{meV}, \\
\Delta E_{1 H F}\left({ }^{1} S_{1 / 2}\right)=-0.0362 \mathrm{meV} .
\end{array}\right.
\end{aligned}
$$

The second-order contribution is given by

$$
\begin{aligned}
E_{2 H F}(S) & =-\frac{2 \mu^{3} \alpha^{4}(1+\kappa)}{3 m_{1} m_{2}}\left\langle\vec{S}_{1} \cdot \vec{S}_{2}\right\rangle \frac{\alpha}{3 \pi} \int_{4}^{\infty} \frac{d x}{x}(1+2 x) \sqrt{1-4 / x} F(\beta \sqrt{x}) \\
& =\left\{\begin{array}{l}
\Delta E_{2 H F}\left({ }^{3} S_{1 / 2}\right)=0.01859 \mathrm{meV}, \\
\Delta E_{2 H F}\left({ }^{1} S_{1 / 2}\right)=-0.05579 \mathrm{meV},
\end{array}\right.
\end{aligned}
$$

where $F(\beta \sqrt{x})$ is given by Eq. (A11). (See [4].) 
For the $p$ state,

$\Delta E_{H F}(P)=-\frac{\mu^{3} \alpha^{4}(1+\kappa)}{6 m_{1} m_{2}} \int_{4}^{\infty} \frac{d x}{x} \frac{\Pi_{e}(x) \beta^{2} x}{(1+\beta \sqrt{x})^{4}}\left\langle\vec{S}_{1} \cdot \vec{S}_{2}\right\rangle$.

Using the expectation values

$$
\begin{aligned}
& \left\langle{ }^{5} P_{3 / 2}\left|\vec{S}_{1} \cdot \vec{S}_{2}\right|{ }^{5} P_{3 / 2}\right\rangle=\frac{1}{4}, \\
& \left\langle{ }^{3} P_{3 / 2}\left|\vec{S}_{1} \cdot \vec{S}_{2}\right|{ }^{3} P_{3 / 2}\right\rangle=-\frac{5}{12}, \\
& \left\langle{ }^{3} P_{1 / 2}\left|\vec{S}_{1} \cdot \vec{S}_{2}\right|{ }^{3} P_{1 / 2}\right\rangle=-\frac{1}{12}, \\
& \left\langle{ }^{1} P_{1 / 2}\left|\vec{S}_{1} \cdot \vec{S}_{2}\right|{ }^{1} P_{1 / 2}\right\rangle=\frac{1}{4},
\end{aligned}
$$

the corresponding energy corrections are

$$
\begin{aligned}
& \Delta E_{H F P}\left({ }^{5} P_{3 / 2}\right)=0.0002 \mathrm{meV}, \\
& \Delta E_{H F P}\left({ }^{3} P_{3 / 2}\right)=-0.0004 \mathrm{meV}, \\
& \Delta E_{H F P}\left({ }^{3} P_{1 / 2}\right)=-0.0001 \mathrm{meV}, \\
& \Delta E_{H F P}\left({ }^{1} P_{1 / 2}\right)=0.0002 \mathrm{meV} .
\end{aligned}
$$

\section{Tensor splitting}

$$
\text { a. } e^{2}(1+\kappa)\left(k_{i} k_{j}-\delta_{i j} \vec{k}^{2} / 3\right) / 4 m_{1} m_{2}
$$

The relevant integral in this case is

$$
\begin{aligned}
& \frac{1}{(2 \pi)^{3}} \int \frac{d k^{3}\left(k_{i} k_{j}-\vec{k}^{2} \delta_{i j} / 3\right) e^{i \vec{k} \cdot \vec{r}}}{\vec{k}^{2}+\lambda} \\
& \quad=\frac{1}{4 \pi r^{3}}\left(\delta_{i j}-3 \hat{x}_{i} \hat{x}_{j}\right)\left(1+\sqrt{\lambda} r+\frac{\lambda r^{2}}{3}\right) e^{-\sqrt{\lambda} r} .
\end{aligned}
$$

(A50a) The expression to be integrated over $\lambda$ is

$$
\begin{aligned}
\left\langle 2 P\left|\Delta V_{T}(\vec{r})\right| 2 P\right\rangle= & \frac{\alpha(1+\kappa)}{m_{1} m_{2}}\left\langle\frac{1}{r^{3}}\left(1+\sqrt{\lambda} r+\frac{\lambda r^{2}}{3}\right) e^{-\sqrt{\lambda} r}\right\rangle \\
& \times\left\langle 3 \vec{S}_{1} \cdot \hat{r} \vec{S}_{2} \cdot \hat{r}-\vec{S}_{1} \cdot \vec{S}_{2}\right\rangle .
\end{aligned}
$$

Now,

$$
\left\langle\frac{1}{r^{3}}\left(1+\sqrt{\lambda} r+\frac{\lambda r^{2}}{3}\right) e^{-\sqrt{\lambda} r}\right\rangle=\frac{1}{24 a^{3}} \frac{\left(1+4 a \sqrt{\lambda}+5 a^{2} \lambda\right)}{(1+a \sqrt{\lambda})^{4}},
$$

so

$$
\left\langle 2 P\left|\Delta V_{T}\right| 2 P\right\rangle=\frac{\mu^{3} \alpha^{5}(1+\kappa)}{72 \pi m_{1} m_{2}} \int_{4}^{\infty} \frac{d x}{x} \frac{(1+2 / x) \sqrt{1-4 / x}\left(1+4 \beta \sqrt{x}+5 \beta^{2} x\right)}{(1+\beta \sqrt{x})^{4}}\left\langle 3 \vec{S}_{1} \cdot \hat{r} \vec{S}_{2} \cdot \hat{r}-\vec{S}_{1} \cdot \vec{S}_{2}\right\rangle
$$

Using

$$
\begin{aligned}
& \left\langle{ }^{5} P_{3 / 2}\left|3 \vec{S}_{1} \cdot \hat{r} \vec{S}_{2} \cdot \hat{r}-\vec{S}_{1} \cdot \vec{S}_{2}\right|{ }^{5} P_{3 / 2}\right\rangle=-\frac{1}{5}, \\
& \left\langle{ }^{3} P_{3 / 2}\left|3 \vec{S}_{1} \cdot \hat{r} \vec{S}_{2} \cdot \hat{r}-\vec{S}_{1} \cdot \vec{S}_{2}\right|{ }^{3} P_{3 / 2}\right\rangle=\frac{1}{6}, \\
& \left\langle{ }^{3} P_{1 / 2}\left|3 \vec{S}_{1} \cdot \hat{r} \vec{S}_{2} \cdot \hat{r}-\vec{S}_{1} \cdot \vec{S}_{2}\right|{ }^{3} P_{1 / 2}\right\rangle=\frac{1}{3}, \\
& \left\langle{ }^{1} P_{1 / 2}\left|3 \vec{S}_{1} \cdot \hat{r} \vec{S}_{2} \cdot \hat{r}-\vec{S}_{1} \cdot \vec{S}_{2}\right|{ }^{1} P_{1 / 2}\right\rangle=-2, \\
& \left\langle{ }^{3} P_{3 / 2}\left|3 \vec{S}_{1} \cdot \hat{r} \vec{S}_{2} \cdot \hat{r}-\vec{S}_{1} \cdot \vec{S}_{2}\right|{ }^{3} P_{1 / 2}\right\rangle=\sqrt{2} / 6,
\end{aligned}
$$

the corrections are

$$
\begin{aligned}
\Delta E_{1 T}\left({ }^{5} P_{3 / 2}\right) & =-0.0003 \mathrm{meV}, \\
\Delta E_{1 T}\left({ }^{3} P_{3 / 2}\right) & =0.0003 \mathrm{meV}, \\
\Delta E_{1 T}\left({ }^{3} P_{1 / 2}\right) & =0.0005 \mathrm{meV}, \\
\Delta E_{1 T}\left({ }^{1} P_{1 / 2}\right) & =-0.0030 \mathrm{meV},
\end{aligned}
$$

$$
\Delta E_{1 T}(\text { Mix })=0.00035 \mathrm{meV}
$$

The perturbative second-order corrections are obtained using Eq. (A38) and

$$
\begin{aligned}
\Delta E_{T}= & -\frac{\mu^{3} \alpha^{5}(1+\kappa)}{36 \pi m_{1} m_{2}} \int_{4}^{\infty} \frac{d x}{x}(1+2 / x) \sqrt{1-4 / x} H(\beta \sqrt{x}) \\
& \times\left\langle 3 \vec{S}_{1} \cdot \hat{r} \vec{S}_{2} \cdot \hat{r}-\vec{S}_{1} \cdot \vec{S}_{2}\right\rangle .
\end{aligned}
$$

The results are

$$
\begin{aligned}
& \Delta E_{2 T}\left({ }^{5} P_{3 / 2}\right)=-0.0001 \mathrm{meV}, \\
& \Delta E_{2 T}\left({ }^{3} P_{3 / 2}\right)=0.0001 \mathrm{meV},
\end{aligned}
$$

$\Delta E_{2 T}\left({ }^{3} P_{1 / 2}\right)=0.0002 \mathrm{meV}$,

$\Delta E_{2 T}\left({ }^{1} P_{1 / 2}\right)=-0.0013 \mathrm{meV}$,

$\Delta E_{1 T}($ Mix $)=0.00015 \mathrm{meV}$. 


\section{APPENDIX B: ONE-LOOP EFFECTIVE POTENTIAL}

To obtain the full one-loop effective potential $V_{4}$, one has to evaluate the one-loop corrections to the single photon exchange potential $V_{2}$ and calculate

$V_{4}(\vec{r})=\frac{1}{(2 \pi)^{3}} \int d^{3} k e^{i \vec{k} \cdot \vec{r}}\left(\sum_{i} V_{4 i}(\vec{k})-\delta\left(V_{2}, V_{2}\right)\right)$,

where $i$ is the sum over all one-loop diagrams and

$$
\begin{aligned}
& \delta\left(V_{2}, V_{2}\right) \\
& =\frac{1}{(2 \pi)^{3}} \int d^{3} p^{\prime \prime} \frac{V_{2}\left(\vec{p}^{\prime}, \vec{p}^{\prime \prime}\right) V_{2}\left(\vec{p}^{\prime \prime}, \vec{p}\right)}{E_{1}(\vec{p})+E_{2}(\vec{p})-E_{1}\left(\vec{p}^{\prime \prime}\right)-E_{2}\left(\vec{p}^{\prime \prime}\right)} .
\end{aligned}
$$

The subtraction is necessary to prevent double counting of the Coulomb exchange in the box diagram.

Using our formulation, the $\alpha^{2} / r^{2}$ term in Eq. (57) arises from the $\delta\left(V_{2}, V_{2}\right)$ subtraction term in Eq. (B1). In momentum space this term behaves as $|\vec{k}|^{-1}$. As can be seen in Eqs. (2.3)-(2.7) of Ref. [14], the only term of this type that survives is the one in $\delta\left(V_{2}, V_{2}\right)$, with $V_{2}\left(\vec{p}^{\prime}, \vec{p}\right)$ given by
$V_{2}\left(\vec{p}^{\prime}, \vec{p}\right)=\frac{-e^{2}}{\left(\vec{p}^{\prime}-\vec{p}\right)^{2}+\lambda^{2}}\left(1-\frac{\left(\vec{p}^{\prime}-\vec{p}\right)^{2}}{8 \mu^{2}}+\frac{\vec{p}^{2}}{m_{1} m_{2}}+\cdots\right)$,

where the dots denote spin-dependent terms that are not relevant.

If one calculates the one-loop effective potential using the Breit-Pauli equation as a starting point, the corresponding subtraction term will involve a $V_{2}\left(\vec{p}^{\prime}, \vec{p}\right)$ of the form

$$
\begin{aligned}
V_{2}\left(\vec{p}^{\prime}, \vec{p}\right)= & \frac{-e^{2}}{\left(\vec{p}^{\prime}-\vec{p}\right)^{2}+\lambda^{2}}\left(1-\frac{1}{8}\left(\frac{1}{m_{1}^{2}}+\frac{1}{m_{2}^{2}}\right)\left(\vec{p}^{\prime}-\vec{p}\right)^{2}\right. \\
& \left.+\frac{\vec{p}^{2}}{m_{1} m_{2}}+\frac{\vec{p} \cdot\left(\vec{p}^{\prime}-\vec{p}\right) \vec{p} \cdot\left(\vec{p}^{\prime}-\vec{p}\right)}{m_{1} m_{2}\left(\vec{p}^{\prime}-\vec{p}\right)^{2}}+\cdots\right) .
\end{aligned}
$$

In this case, the last term in Eq. (B4) exactly cancels the coefficient of the $|\vec{k}|^{-1}$ term produced by the second and third terms when $\delta\left(V_{2}, V_{2}\right)$ is evaluated. Consequently, there is no $\alpha^{2} / r^{2}$ term in the one-loop corrections to the Breit-Pauli potential.
[1] R. Pohl et al., Nature (London) 466, 213 (2010).

[2] A. Antognini et al., Science 339, 417 (2013).

[3] E. Borie and G. A. Rinker, Rev. Mod. Phys. 54, 67 (1982).

[4] E. Borie, Phys. Rev. A 71, 032508 (2005).

[5] K. Pachucki, Phys. Rev. A 53, 2092 (1996).

[6] K. Pachucki, Phys. Rev. A 60, 3593 (1999).

[7] R. Pohl, R. Gilman, G. A. Miller, and K. Pachucki, Annu. Rev. Nucl. Part. Sci. 63, 175 (2013).

[8] A. Antognini, F. Kottmann, F. Biraben, P. Indelicato, F. Nez, and R. Pohl, Ann. Phys. (Amsterdam) 331, 127 (2013).

[9] C. Peset and A. Pineda, Eur. Phys. J. A 51, 156 (2015).

[10] P. J. Mohr, B. N. Taylor, and D. B. Newell, Rev. Mod. Phys. 84, 1527 (2012), and Ref. [9].

[11] https://www.jlab.org/prad/collaboration.html.

[12] A. Beyer et al., Science 358, 79 (2017).

[13] H. Fleurbaey, S. Galtier, S. Thomas, M. Bonnaud, L. Julien, F. Biraben, F. Nez, M. Abgrall, and J. Guéna, Phys. Rev. Lett. 120, 183001 (2018).

[14] S. N. Gupta, W. W. Repko, and C. J. Suchyta III, Phys. Rev. D 40, 4100 (1989).

[15] See the related work: S. N. Gupta, Nucl. Phys. 57, 19 (1964); S. N. Gupta and S. F. Radford, Phys. Rev. D 21, 2213 (1980).
[16] K. A. Olive et al. (Particle Data Group), Chin. Phys. C 38, 090001 (2014).

[17] G. Källen, Helv. Phys. Acta 25, 417 (1952).

[18] H. Lehmann, Nuovo Cimento 11, 342 (1954).

[19] G. Källen and A. Sabry, Dan. Mat. Fys. Medd. 29, 1 (1955).

[20] J. Blomqvist, Nucl. Phys. B48, 95 (1972).

[21] T. Kinoshita and M. Nio, Phys. Rev. Lett. 82, 3240 (1999).

[22] L. Hostler and R. H. Pratt, Phys. Rev. Lett. 10, 469 (1963).

[23] L. Hostler, Phys. Rev. 178, 126 (1969); J. Math. Phys. 16, 1585 (1975).

[24] B. J. Laurenzi and A. Flamberg, Int. J. Quantum Chem. XI, 869 (1971).

[25] B. R. Johnson and J. O. Hirschfelder, J. Math. Phys. 20, 2484 (1979).

[26] V. G. Ivanov, E. Y. Korzinin, and S. G. Karshenboim, Phys. Rev. D 80, 027702 (2009).

[27] G. W. Erickson and D. R. Yennie, Ann. Phys. (N.Y.) 35, 271 (1965).

[28] M. E. Rose, Relativistic Electron Theory (Wiley, New York, 1961), p. 178.

[29] U. D. Jentschura, Phys. Rev. A 84, 012505 (2011). 